\title{
On the definition of strange nonchaotic attractor
}

\author{
by \\ Lluís Alsedà and Sara Costa (Barcelona) \\ Dedicated to Michat Misiurewicz on the occasion of his 60th birthday
}

\begin{abstract}
The aim of this paper is twofold. On the one hand, we want to discuss some methodological issues related to the notion of strange nonchaotic attractor. On the other hand, we want to formulate a precise definition of this kind of attractor, which is "observable" in the physical sense and, in the two-dimensional setting, includes the well known models proposed by Grebogi et al. and by Keller, and a wide range of other examples proposed in the literature. Furthermore, we analytically prove that a whole family of two-dimensional quasiperiodic skew products defined on $\mathbb{S}^{1} \times \mathbb{R}$ have strange nonchaotic attractors. As a corollary we show analytically that the system proposed by Grebogi et al. has a strange nonchaotic attractor.
\end{abstract}

1. Introduction. The notion of strange nonchaotic attractor (briefly SNA) was introduced $\left({ }^{1}\right)$ by Grebogi et al. in [10] when studying attractors of quasiperiodically forced skew products of the form

$$
\left\{\begin{array}{l}
\theta_{n+1}=\theta_{n}+\omega(\bmod 1) \\
x_{n+1}=\psi\left(\theta_{n}, x_{n}\right)
\end{array}\right.
$$

where $x \in \mathbb{R}, \theta \in \mathbb{S}^{1}$ and $\omega \in \mathbb{R} \backslash \mathbb{Q}$. One of the two examples they considered consisted in taking $\psi(\theta, x)=2 \sigma \cos (2 \pi \theta) \tanh (x)$ in the above system (see Figure 1 for a picture of the attractor of an instance of this system):

$$
\left\{\begin{array}{l}
\theta_{n+1}=\theta_{n}+\omega(\bmod 1) \\
x_{n+1}=2 \sigma \cos \left(2 \pi \theta_{n}\right) \tanh \left(x_{n}\right)
\end{array}\right.
$$

2010 Mathematics Subject Classification: Primary 37C55, 34D08, 37C70.

Key words and phrases: quasiperiodically forced system, strange nonchaotic attractor, Lyapunov exponents.

$\left({ }^{1}\right)$ This kind of attractor had already been studied in the literature much earlier than the term SNA was coined. For example in $[18,19,24]$ constructions of flows containing strange nonchaotic attractors can be found. Also, in the last part of this paper, we see that some well known one-dimensional attractors are SNA's. 


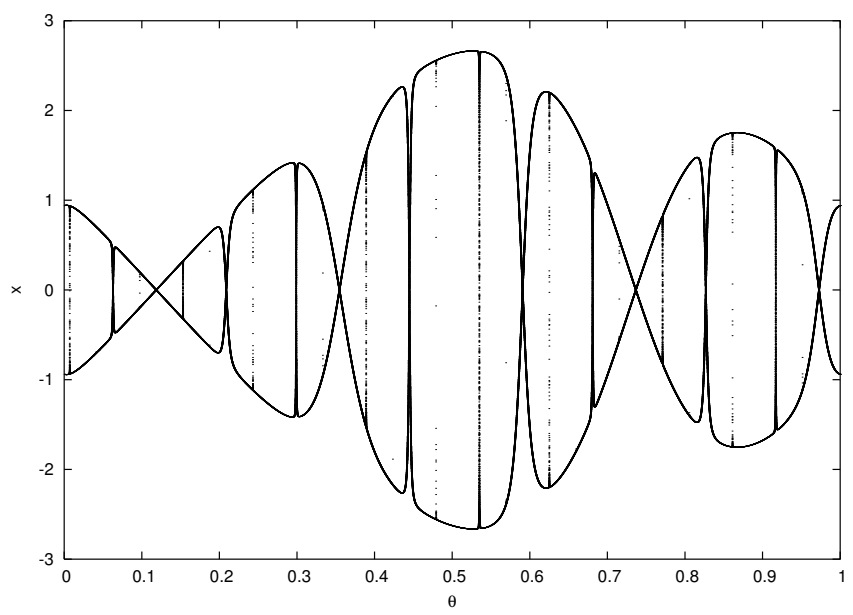

Fig. 1. The attractor of (2) with $\sigma=1.5$

The authors of [10] defined an SNA as an attractor whose Lyapunov exponents are nonpositive almost everywhere and which is neither finite nor piecewise differentiable. An attractor in this definition is a compact set with a neighbourhood such that, for almost every initial condition in this neighbourhood, the limit set of the orbit as time tends to $+\infty$ is in the attractor. An attractor is piecewise differentiable if it is either a piecewise differentiable curve or surface, or a volume bounded by a piecewise differentiable closed surface. Later on, it was proved by G. Keller [17] for systems similar to (2) that the attractor cuts the repeller $x=0$ in an invariant set which is dense (in $x=0$ ) and it is different from zero in a set whose projection onto $\mathbb{S}^{1}$ has full measure. This gives the strangeness of such attractors.

As already explained in [10], in this theory, the term chaotic refers to the dynamics on the attractor, while strange refers to the geometrical structure of the attractor.

There is a lot of controversy about what should be the precise definition of SNA and whether the elements involved must be "observable" in a physical and computational sense. Indeed, this notion is often not even precisely formulated. On the other hand, unfortunately, one can find much more empirical and crude numerical studies about SNA's than rigorous proofs of their existence, and there are also some theoretical issues about a number of the numerical studies. Some remarkable examples of rigorous studies of the existence of such attractors can be found in several contexts. For example, system (2) is studied in [4]; [1] and [5] are devoted to the study of the quasiperiodic quadratic family; in [14] the quasiperiodic Arnold map is considered; in [13], $S L(2, \mathbb{R})$ cocycles are studied; [12] is devoted to the study of 
Harper maps; and, finally, in [11] and [17] the authors consider a quasiperiodically forced system of the form (1) defined on the positive semi-cylinder $\mathbb{S}^{1} \times[0, \infty)$, where the second component is given by $\psi(\theta, x)=g(\theta) f(x)$ and $f$ is monotonic.

The aim of this paper is to give a rigorous definition of SNA in the topological setting, while trying to clarify and fix the above mentioned theoretical issues. This will be done in the third section.

The second section is devoted to introducing the notation used along this paper and to showing two paradigmatic examples in this field. In the third section, as we have said, we propose a rigorous definition and discuss some methodological aspects relating to the nonchaoticity part of the definition. This section is the core of this paper. Finally, in the last section we check that the two examples given in the second section are SNA's with the definition we propose. Also, we prove that two well known kinds of attractor in onedimensional dynamics, the absorbing Cantor set and the solenoidal attractor, are SNA's.

2. Notation and motivating examples. We are interested in twodimensional quasiperiodically forced skew products. These are systems defined on the Cartesian product of the circle $\mathbb{S}^{1}=\mathbb{R} / \mathbb{Z}$ and a metric space $X$, of the form

$$
\left\{\begin{array}{l}
\theta_{n+1}=R_{\omega}\left(\theta_{n}\right)=\theta_{n}+\omega(\bmod 1) \\
x_{n+1}=\psi\left(\theta_{n}, x_{n}\right)
\end{array}\right.
$$

where $\left(\theta_{n}, x_{n}\right) \in \mathbb{S}^{1} \times X$ and $\omega \in \mathbb{R} \backslash \mathbb{Q}$. The first component, which is independent of the second, is the basis of the skew product. These systems map each fibre $\{\theta\} \times X$ into another one $\left\{R_{\omega}(\theta)\right\} \times X$.

Recall that $R_{\omega}$ is minimal and has the Lebesgue measure as a unique invariant measure (thus being uniquely ergodic). Moreover, every invariant attracting set is the graph of a correspondence $\varphi$ from $\Lambda$ to $X$, where $\Lambda$ is an invariant set of $R_{\omega}$ of full Lebesgue measure. Then $\varphi$ must satisfy the invariance equation

$$
\varphi\left(R_{\omega}(\theta)\right)=\psi(\theta, \varphi(\theta))
$$

for every $\theta \in \Lambda$, or equivalently, it must be a fixed point of the transfer operator defined by

$$
\mathcal{T} \varphi(\theta):=\psi\left(\theta, \varphi\left(R_{\omega}^{-1}(\theta)\right)\right) .
$$

Note that since $\varphi$ is a correspondence, the equality in the above formula is between sets.

EXAMPLE 1 (Grebogi et al. [10]). In this example we discuss the study of system (2) done in [10], together with the proposed notion of SNA. We 
recall that the system considered is

$$
\left\{\begin{array}{l}
\theta_{n+1}=R_{\omega}\left(\theta_{n}\right), \\
x_{n+1}=2 \sigma \tanh \left(x_{n}\right) \cos \left(2 \pi \theta_{n}\right),
\end{array}\right.
$$

where $\left(\theta_{n}, x_{n}\right) \in \mathbb{S}^{1} \times \mathbb{R}$ and $\omega \in \mathbb{R} \backslash \mathbb{Q}$. For every $\left(\theta_{0}, x_{0}\right)$ and $n \in \mathbb{N}$ it follows that $\left(\theta_{n}, x_{n}\right) \in \mathbb{S}^{1} \times[-2 \sigma, 2 \sigma]$. So, the system has an attractor in this region. As mentioned before, attractor in [10] means a compact set with a neighbourhood such that, for almost every initial condition in this neighbourhood, the $\omega$-limit set of the orbit is in the attractor. Observe that this definition is very similar to the one proposed by Milnor [20].

The attractor is the closure of the graph of a map $\varphi: \mathbb{S}^{1} \rightarrow[-2 \sigma, 2 \sigma] \subset \mathbb{R}$ and, as we already know, it must satisfy the invariance equation

$$
\varphi\left(R_{\omega}(\theta)\right)=2 \sigma \cos (\theta) \tanh (\varphi(\theta)) .
$$

Since $\tanh (0)=0$, the circle $x=0$ is invariant. Thus, by the Birkhoff Ergodic Theorem, it can be shown that the vertical Lyapunov exponent at $x=0$ is

$$
\int_{\mathbb{S}^{1}} \log \left|\frac{\partial(2 \sigma \tanh (x) \cos (2 \pi \theta))}{\partial x}\right|_{x=0}|d \theta=\log | \sigma \mid
$$

for almost every $\theta \in \mathbb{S}^{1}$ with respect to the Lebesgue measure. So, if $|\sigma|>1$, the vertical Lyapunov exponent at $x=0$ is positive and the circle $x=0$ is a repeller. Consequently, the map $\varphi$ is not identically zero. Moreover, since $\cos (2 \pi \theta)$ vanishes for $\theta \in\{1 / 4,3 / 4\}$ it follows that the set

$$
D:=\{(i / 4+n \omega(\bmod 1), 0): n \in \mathbb{Z}, i \in\{1,3\}\}
$$

is invariant and dense in $x=0$, and belongs at the same time to the repeller and to the attractor. The authors use numerical techniques to find that $\varphi$ is positive almost everywhere, and hence discontinuous almost everywhere. This gives the strangeness of the attractor.

The nonchaoticity is studied in terms of Lyapunov exponents. An attractor is defined to be nonchaotic whenever the Lyapunov exponents are nonpositive for almost every point in the basin of attraction of the attractor (that is, in the set of initial conditions which approach the attractor as time tends to $+\infty)$. For this kind of system the vertical Lyapunov exponent at $(\theta, x)$ is given by

$$
\limsup _{n \rightarrow \infty} \frac{1}{n} \log \left\|\left(\begin{array}{ll}
\partial \theta_{n} / \partial \theta & \partial \theta_{n} / \partial x \\
\partial x_{n} / \partial \theta & \partial x_{n} / \partial x
\end{array}\right)\left(\begin{array}{l}
0 \\
1
\end{array}\right)\right\|=\limsup _{n \rightarrow \infty} \frac{1}{n} \log \left|\frac{\partial x_{n}}{\partial x}\right| .
$$

By Oseledets's Theorem, $\lim _{n \rightarrow \infty} n^{-1} \log \left|\partial x_{n} / \partial x\right|$ exists for almost every point in the support of any invariant measure and, as we will see, the other Lyapunov exponent is zero. 
Using the geometrical properties of the hyperbolic tangent function it can be easily proved that $\left|\tanh ^{\prime}(x)\right|<\tanh (x) / x$; this is used by the authors to check that, whenever the above limit exists, the vertical Lyapunov exponent is negative. This provides a rigorous proof of the nonpositivity of the Lyapunov exponent for almost every point with respect to an invariant measure but in general it does not control the value of the Lyapunov exponent for Lebesgue almost every point in the basin of attraction of the attractor.

ExAmple 2 (Keller [17]). As far as we know, [17] together with [4] contain the first rigorous proofs of the existence of such SNA.

Keller proved the existence of an SNA (with the definition used in [10]) for two-dimensional quasiperiodically forced skew products of the form

$$
\left\{\begin{array}{l}
\theta_{n+1}=R_{\omega}\left(\theta_{n}\right), \\
x_{n+1}=f\left(x_{n}\right) g\left(\theta_{n}\right),
\end{array}\right.
$$

where $\left(\theta_{n}, x_{n}\right) \in \mathbb{S}^{1} \times[0, \infty)$, the map $g: \mathbb{S}^{1} \rightarrow[0, \infty)$ is continuous and bounded, and the map $f:[0, \infty) \rightarrow[0, \infty)$ is $\mathcal{C}^{1}$, bounded, increasing, strictly concave and such that $f(0)=0$. Such models are "one-sided" generalisations of (2). To see this and to fix ideas, notice that $f(x)$ can be taken equal to $\tanh (x)$ and $g(x)$ equal to $2|\sigma \cos (2 \pi \theta)|$.

Set

$$
\mathcal{I}(g):=\int_{\mathbb{S}^{1}} \log g(\theta) d \theta \geq-\infty,
$$

which is well defined because $g$ is bounded. As in system (2), the circle $x=0$ is invariant and the vertical Lyapunov exponent on this invariant set is the logarithm of

$$
\sigma:= \begin{cases}f^{\prime}(0) \exp (\mathcal{I}(g)) & \text { if } \mathcal{I}(g)>-\infty \\ 0 & \text { otherwise, }\end{cases}
$$

for almost every $\theta \in \mathbb{S}^{1}$. So, if $\sigma>1$, the circle $x=0$ is a repeller.

Then the attractor and its dynamics are described by the following

TheOREM 2.1 (Keller). There exists an upper semicontinuous map $\varphi$ : $\mathbb{S}^{1} \rightarrow \mathbb{R}^{+}$whose graph is invariant under system (6). Moreover:

(a) the Lebesgue measure on the circle, lifted to the graph of $\varphi$, is a Sinai-Ruelle-Bowen measure, that is,

$$
\lim _{n \rightarrow \infty} \frac{1}{n} \sum_{k=0}^{n-1} \vartheta\left(F^{k}(\theta, x)\right)=\int_{\mathbb{S}^{1}} \vartheta(\theta, \varphi(\theta)) d \theta
$$

for every $\vartheta \in \mathcal{C}^{0}\left(\mathbb{S}^{1} \times \mathbb{R}^{+}, \mathbb{R}\right)$ and Lebesgue almost every $(\theta, x) \in$ $\mathbb{S}^{1} \times \mathbb{R}^{+}$, where $F$ denotes the map $(\theta, x) \mapsto\left(R_{\omega}(\theta), f(x) g(\theta)\right)$,

(b) if $\sigma \leq 1$ then $\varphi \equiv 0$,

(c) if $\sigma>1$ then $\varphi(\theta)>0$ for almost every $\theta$, 
(d) if $\sigma>1$ and $g\left(\theta_{0}\right)=0$ for some $\theta_{0}$ then the set $\{\theta: \varphi(\theta)>0\}$ is meager and $\varphi$ is almost everywhere discontinuous,

(e) if $\sigma>1$ and $g>0$ then $\varphi$ is positive and continuous; if $g$ is $\mathcal{C}^{1}$ then so is $\varphi$,

(f) if $\sigma \neq 1$ then $\left|x_{n}-\varphi\left(\theta_{n}\right)\right| \rightarrow 0$ exponentially fast for almost every $\theta$ and every $x>0$.

When $\sigma>1$ it follows that the vertical Lyapunov exponent exists for almost every $\theta$ and for every $x>0$ and it is equal to

$$
\mathcal{I}(g)+\int_{\mathbb{S}^{1}} \log f^{\prime}(\varphi(\theta)) d \theta<0 .
$$

By using Oseledets's Theorem it can be shown that the second Lyapunov exponent is zero for every $(\theta, x)$ such that the vertical Lyapunov exponent exists with limit. Hence, case (d) of the above theorem shows the existence of SNA for such systems.

Let us point out that the graph of $\varphi$ is not closed because it is discontinuous almost everywhere. Therefore the attractor must be the closure of this graph.

3. A definition of strange nonchaotic attractor. As we have said in the introduction, there is no unique notion of SNA in the literature. Moreover, depending on the definition chosen, certain objects will be or will not be SNA's and the difficulty of their study can vary. The aim of this section is to give a rigorous definition of this kind of attractor which supports theoretically the SNA's found in the above models. We also look for a definition that agrees with the numerical approach in the quest for SNA's. Indeed, we want a definition whose elements are "observable" in the physical sense (that is, which involves sets of positive Lebesgue measure).

We divide this section into four subsections. In the first three of them we will define one of the terms involved in the definition of SNA: attractor, nonchaotic and strange. In the last one we will state a common definition of SNA in the literature (that we call the one-dimensional approach) and we will compare it with the definition we propose.

3.1. Attractor. We adopt the definition of attractor proposed by Milnor in [20]:

Definition 3.1. Let $(X, f)$ be a (semi-)dynamical system where $X$ is a smooth compact manifold endowed with a measure $\mu$ equivalent to the Lebesgue one when it is restricted to any coordinate neighbourhood and $f$ is a continuous map. A closed subset $\mathcal{A} \subset X$ will be called an attractor if it satisfies the following two conditions:

(I) The set $\rho(\mathcal{A}):=\{z \in X: \omega(z) \subset \mathcal{A}\}$ has strictly positive measure. 
(II) There is no strictly smaller closed set $\mathcal{A}^{\prime} \subset \mathcal{A}$ so that $\rho\left(\mathcal{A}^{\prime}\right)$ coincides with $\rho(\mathcal{A})$ up to a set of measure zero.

The set $\rho(\mathcal{A})$ is called the realm of attraction of $\mathcal{A}$, and it can be defined for every subset of $X$. When it is open, it is called the basin of attraction of $\mathcal{A}$. A set satisfying this definition is called a metric attractor.

Any attractor $\mathcal{A}$ must be a nonempty closed $f$-invariant (i.e. $f(\mathcal{A})=\mathcal{A}$ ) subset of the nonwandering set of $f$.

Notice that the realm of attraction of a repeller has measure zero. Thus, an attractor $\mathcal{A}$ may contain a repeller. Also, it may contain a smaller attractor $\mathcal{A}^{\prime} \subsetneq \mathcal{A}$ as long as $\rho(\mathcal{A})$ and $\rho\left(\mathcal{A}^{\prime}\right)$ differ by a set of positive measure. The attractors for which this is not possible are minimal attractors. They are defined by replacing condition (II) of Definition 3.1 by

(II') There is no strictly smaller closed set $\mathcal{A}^{\prime} \subset \mathcal{A}$ such that $\mu\left(\rho\left(\mathcal{A}^{\prime}\right)\right)$ is positive.

If the space $X$ is not compact but there exists a compact set $\widetilde{X}$ with positive measure such that $f(\widetilde{X}) \subset \widetilde{X}$, then there exists an attractor in $\widetilde{X}$.

There is an important choice to make for the definition of attractor. Either we require the attractor to be closed and then, in the examples considered, the repeller $x=0$ is contained in the attractor, or we omit this condition. In that case, the $\omega$-limit sets of points in the attractor need not be contained in it, and this causes invariance problems. We have chosen to define an attractor as a closed set.

3.2. Strangeness. Concerning SNA's we have found three different definitions of strangeness. In the literature, an attractor is considered strange in the following cases:

(1) It is neither a finite set of points nor piecewise differentiable (see, for instance, [10]).

(2) It has fractal geometry (that is, its Hausdorff dimension is greater than its topological dimension - see, for instance, [22]).

(3) Its Hausdorff dimension is greater than one (see, for instance, [21]).

As far as we know, the three definitions are used in articles where twodimensional systems are studied, while for higher-dimensional systems only the first one is used.

It is an exercise to show that the Hausdorff dimension of the graph of a one-dimensional piecewise differentiable map is one. Thus, one can show that, in the two-dimensional case, the third definition implies the second one and, in turn, this implies the first one. So we choose as a definition of strangeness the more general one: 
Definition 3.2. An attractor is strange if it is neither a finite set of points nor a piecewise differentiable manifold. A manifold $X$ is piecewise differentiable if there exists a finite set of disjoint differentiable submanifolds $A_{1}, \ldots, A_{k}$ such that

$$
X \subset \mathrm{Cl}\left(\bigcup_{i=1}^{k} A_{i}\right) .
$$

If $\partial X \neq \emptyset$, then the boundary is also required to be piecewise differentiable.

3.3. Nonchaoticity. As is customary in the world of SNA's, we define the notion of nonchaotic in terms of Lyapunov exponents.

Let $(X, f)$ be a (semi-)dynamical system where $X$ is a finite-dimensional, smooth, compact, Riemannian manifold. The maximal upper Lyapunov exponent of $x \in X$ is defined as

$$
\begin{aligned}
\lambda_{\max }(x) & =\limsup _{n \rightarrow \infty} \frac{1}{n} \log \left|D f^{n}(x)\right| \\
& =\max \left\{\limsup _{n \rightarrow \infty} \frac{1}{n} \log \left\|D f^{n}(x) v\right\|: v \in T_{x} X \backslash\{0\}\right\} .
\end{aligned}
$$

Observe that the maximal upper Lyapunov exponent always exists. Moreover, since $X$ is finite-dimensional, its value does not depend on the choice of the compatible matrix norm. Also, by Oseledets's Theorem, it exists in terms of limits for almost every point in the support of any invariant measure.

Definition 3.3. Let $(X, f)$ be a (semi-)dynamical system where $X$ is a finite-dimensional, smooth, compact, Riemannian manifold. An attractor $\mathcal{A}$ is called nonchaotic if the set of points $x \in \rho(\mathcal{A})$ for which $\lambda_{\max }(x)>0$ has zero Lebesgue measure.

This definition implies that the maximal upper Lyapunov exponent is non-positive for almost every point in $\rho(\mathcal{A})$, which has positive Lebesgue measure. Thus, in contrast with other definitions of nonchaoticity used in the literature (that only take into account sets of Lebesgue measure zero) the above definition depends on quantities (limsup) that are well defined for every point, and properties $\left(\lambda_{\max }(x) \leq 0\right)$ that must be satisfied in sets of positive Lebesgue measure, thus being observable.

Another approach to the definition of nonchaoticity for systems of the form (1) is (see for instance [15], [16]) to consider the dynamical system in dimension one restricted to the attractor. Then the original system is called nonchaotic if the unique Lyapunov exponent of this reduced system is nonpositive. The Lyapunov exponent is defined (with lim) for almost every point in the attractor by the Birkhoff Ergodic Theorem since the dynamics on the attractor is driven by $\theta_{n+1}=R_{\omega}\left(\theta_{n}\right)$, which is uniquely ergodic with the unique ergodic measure being the Lebesgue measure. The drawback of 
this definition, in our opinion, is that the nonchaoticity condition is not observable in the above sense.

Rather often in the literature, Lyapunov exponents are computed (frequently in a very crude numerical way) for Lebesgue almost every point and with lim instead of limsup without checking the existence of such numbers. To see the problems that can arise from this approach, in the next section we discuss some theoretical issues about Lyapunov exponents and provide two examples showing that, in general, these problems have to be taken into account.

3.3.1. Comments on the use of Lyapunov exponents in the definition of nonchaoticity. Let $X$ be an $m$-dimensional manifold, let $F$ be a differentiable map from $X$ to itself, and let $\mu$ be an $F$-invariant measure. Then by Oseledets's Theorem there exist $m$ Lyapunov exponents, $-\infty \leq \lambda_{1} \leq \cdots \leq$ $\lambda_{m}<\infty$, such that for $\mu$-almost every $x \in X$ it follows that

$$
\lim _{n \rightarrow \infty} \frac{1}{n} \log \left\|D F^{n}(x) v\right\| \in\left\{\lambda_{1}, \ldots, \lambda_{m}\right\}
$$

for every $v$ in the tangent space to $X$ at $x$, and

$$
\lim _{n \rightarrow \infty} \frac{1}{n} \log \left|\operatorname{det} D F^{n}(x)\right|=\sum_{i=1}^{m} \lambda_{i} .
$$

The points which satisfy the above conditions are called regular points.

Consequently, in the case of two-dimensional skew products of the form (1), for $\mu$-almost every $(\theta, x)$, the Lyapunov exponents are the vertical Lyapunov exponent:

$$
\lambda_{v}=\lim _{n \rightarrow \infty} \frac{1}{n} \log \left\|\left(\begin{array}{cc}
1 & 0 \\
\partial x_{n} / \partial \theta & \partial x_{n} / \partial x
\end{array}\right)\left(\begin{array}{l}
0 \\
1
\end{array}\right)\right\|=\lim _{n \rightarrow \infty} \frac{1}{n} \log \left|\frac{\partial x_{n}}{\partial x}\right|,
$$

and a second one that we will denote by $\widehat{\lambda}$. Moreover, from (8) it follows that, for $\mu$-almost every $(\theta, x)$,

$$
\lambda_{v}+\hat{\lambda}=\lim _{n \rightarrow \infty} \frac{1}{n} \log \left|\operatorname{det}\left(\begin{array}{cc}
1 & 0 \\
\partial x_{n} / \partial \theta & \partial x_{n} / \partial x
\end{array}\right)\right|=\lim _{n \rightarrow \infty} \frac{1}{n} \log \left|\frac{\partial x_{n}}{\partial x}\right|=\lambda_{v} .
$$

We can summarize the above arguments as:

REMARK 3.4. The second Lyapunov exponent $\widehat{\lambda}$ is zero, and the maximal Lyapunov exponent $\lambda_{\max }=\max \left\{\lambda_{v}, \widehat{\lambda}\right\}$ is nonpositive if and only if $\lambda_{v} \leq 0$.

It is also important to point out that the above formulas do not work for arbitrary points outside the support of an invariant measure. Then the Lyapunov exponents are only defined in terms of limsup. In [3] it is shown that the set of points whose Lyapunov exponents are not given in terms of limits may be nonempty and have full Hausdorff dimension (but it has 
zero measure with respect to any invariant measure). Moreover, property (8) does not necessarily hold after replacing lim by limsup. A nice and simple example of this fact, due to R. de la Llave [8], is described in Example 3. We should note that, in view of Oseledets's Theorem, the set of points for which (8) does not hold has zero measure with respect to any invariant measure.

Example 3. The system we consider is a nonsymmetric horseshoe. To define it in detail we follow Robinson's construction of the horseshoe map [23].
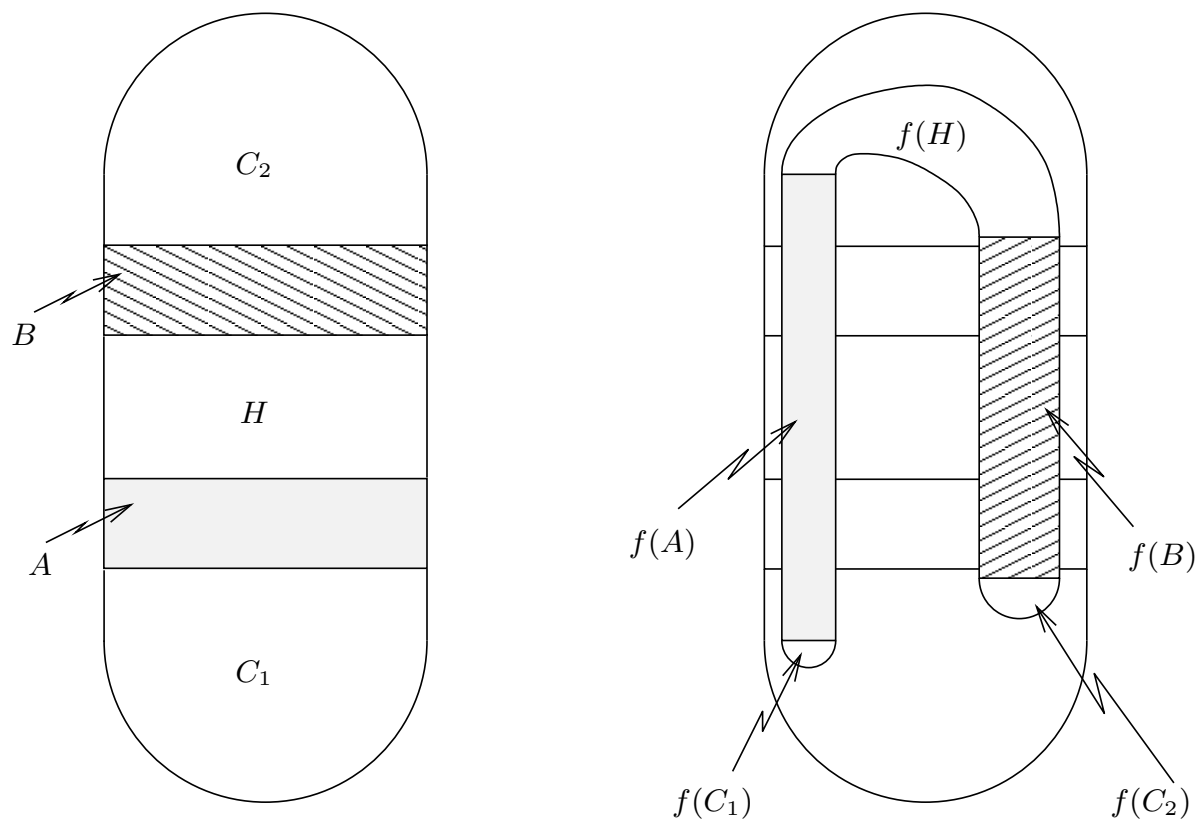

Fig. 2. Construction of the sets $N$ and $f(N)$

We start by taking $\lambda<1 / 2$ and the unit square divided into three horizontal strips (see Figure 2),

$$
[0,1] \times[0,1]=A \cup H \cup B,
$$

where $A:=[0,1] \times[0, \lambda]$ and $B:=[0,1] \times[1-\lambda, 1]$. Let $f \in \mathcal{C}^{\infty}([0,1] \times[0,1])$ be such that

$$
\left.D f\right|_{A}=\left(\begin{array}{cc}
d / \kappa \alpha & 0 \\
0 & \kappa \alpha
\end{array}\right) \text { and }\left.D f\right|_{B}=\left(\begin{array}{cc}
-d / \alpha & 0 \\
0 & -\alpha
\end{array}\right),
$$

with $\kappa>1, d, \alpha>0,1 / \alpha<\lambda<1 / 2$ and $0<d / \kappa \alpha+d / \alpha<1$. Therefore,

$$
\kappa \alpha>\alpha \text { and } \frac{d}{\kappa \alpha}<\frac{d}{\alpha} .
$$


Now we set $N:=([0,1] \times[0,1]) \cup C_{1} \cup C_{2}$, where $C_{1}=\left([0,1] \times\left[-\frac{\kappa \alpha \lambda-1}{2}, 0\right]\right) \cup S_{1}, \quad C_{2}=\left([0,1] \times\left[1,1+\frac{\kappa \alpha \lambda-1}{2}\right]\right) \cup S_{2}$, and $S_{1}$ and $S_{2}$ are the semidiscs of radius $1 / 2$ on the bottom of $[0,1] \times$ $[-(\kappa \alpha \lambda-1) / 2,0]$ and on the top of $[0,1] \times[1,1+(\kappa \alpha \lambda-1) / 2]$, respectively.

Finally, we can extend $f$ to a $\mathcal{C}^{\infty}$ diffeomorphism of $N$ in such a way that $f(H) \subset C_{2}, f\left(C_{2}\right) \subset C_{1}, f\left(C_{1}\right) \subset C_{1}$ and $\left.f\right|_{C_{1}}$ is contractive, so that $f$ has a unique fixed point which is contained in $f\left(C_{1}\right)$.

Associated to this diffeomorphism we can consider a symbolic dynamics analogous to the one associated to Smale's horseshoe.

It is well known that the unique invariant set of this map contained in the unit square is

$$
\Lambda:=\left\{x \in[0,1] \times[0,1]: f^{i}(x) \in A \cup B \text { for all } i \in \mathbb{Z}\right\} .
$$

We consider the set $\widetilde{\Lambda}$ of those points $x \in \Lambda$ such that, for some $l \in$ $\mathbb{N} \cup\{0\}$, the itinerary of $f^{l}(x)$ is of the form

$$
A^{p_{1}} B^{p_{2}-p_{1}} A^{p_{3}-p_{2}} B^{p_{4}-p_{3}} \cdots A^{p_{2 k+1}-p_{2 k}} B^{p_{2 k+2}-p_{2 k+1}} \cdots
$$

where $p_{k}=\rho^{k}$ with $\rho \in \mathbb{N} \backslash\{1\}$. We set $p_{0}=0$. Then, for every $n \in$ $\left[p_{k}, p_{k+1}-1\right]$,

$$
f^{l+n}(x) \in \begin{cases}A & \text { if } k \text { is even } \\ B & \text { if } k \text { is odd }\end{cases}
$$

Clearly, $f(\widetilde{\Lambda}) \subset \widetilde{\Lambda}$.

The next result summarises the situation for the Lyapunov exponents of the above model and, in particular, shows that condition (8) does not hold for this example.

Lemma 3.5. For every point $x \in \widetilde{\Lambda}$, the upper Lyapunov exponents are

$$
\begin{aligned}
& \limsup _{n \rightarrow \infty} \frac{1}{n} \log \left\|D f^{n} x\left(\begin{array}{l}
1 \\
0
\end{array}\right)\right\|=\log (d / \alpha), \\
& \limsup _{n \rightarrow \infty} \frac{1}{n} \log \left\|D f^{n} x\left(\begin{array}{l}
0 \\
1
\end{array}\right)\right\|=\log (\kappa \alpha) .
\end{aligned}
$$

Moreover,

$$
\limsup _{n \rightarrow \infty} \frac{1}{n} \log \left|\operatorname{det}\left(D f^{n} x\right)\right|=\lim _{n \rightarrow \infty} \frac{1}{n} \log \left|\operatorname{det}\left(D f^{n} x\right)\right|=\log (d) .
$$

Therefore, since $\kappa>1$, formula (8) does not hold.

Proof. Since we have to take limits, we can skip the first iterates of $x$ and assume that $x$ already has an itinerary of the form (11) (that is, we can assume that $l=0)$. 
We start by proving that the upper Lyapunov exponent in the direction $(1,0)$ is $\log (d / \alpha)$. Without loss of generality we can work with $\|\cdot\|_{\infty}$, because the value of the Lyapunov exponents does not depend on the chosen norm. Given $n \in \mathbb{N}$ we set

$$
K(n):=\frac{1}{n} \log \left\|D f^{n} x\left(\begin{array}{l}
1 \\
0
\end{array}\right)\right\|_{\infty}=\frac{m_{1} \log (d / \kappa \alpha)+m_{2} \log (d / \alpha)}{m_{1}+m_{2}},
$$

where $n=m_{1}+m_{2}$, and $m_{1}=m_{1}(n)$ and $m_{2}=m_{2}(n)$ are the numbers of iterates of $x$ which are in $A$ and $B$, respectively. Since $m_{1} \geq 0$ and $m_{2} \geq 0$ we have

$$
\log (d / \kappa \alpha) \leq K(n) \leq \log (d / \alpha) .
$$

On the other hand,

$$
\begin{aligned}
\limsup _{n \rightarrow \infty} K(n) & =\limsup _{n \rightarrow \infty} \frac{m_{1} \log (d / \kappa \alpha)+m_{2} \log (d / \alpha)}{m_{1}+m_{2}} \\
& =\limsup _{n \rightarrow \infty} \frac{m_{2}(\log (d / \alpha)-\log (d / \kappa \alpha))+\left(m_{1}+m_{2}\right) \log (d / \kappa \alpha)}{n} \\
& =\log (d / \kappa \alpha)+(\log (d / \alpha)-\log (d / \kappa \alpha)) \limsup _{n \rightarrow \infty} \frac{m_{2}}{n} \leq \log (d / \alpha) .
\end{aligned}
$$

Thus, to prove that $\lim \sup _{n \rightarrow \infty} K(n)=\log (d / \alpha)$ it is enough to show that there exists a sequence $\left\{n_{k}\right\}_{k \in \mathbb{N}} \subset \mathbb{N}$ such that

$$
\lim _{k \rightarrow \infty} \frac{m_{2}\left(n_{k}\right)}{n_{k}}=1 .
$$

We set $n_{k}=p_{2 k}-1=\rho^{2 k}-1$. Then, in view of (11),

$m_{2}\left(n_{k}\right)=\sum_{i=1}^{k}\left(p_{2 i}-p_{2 i-1}\right)=\sum_{i=1}^{k}\left(\rho^{2 i}-\rho^{2 i-1}\right)=\frac{\rho-1}{\rho} \frac{\rho^{2}-\rho^{2 k}}{1-\rho^{2}}=\frac{\rho^{2 k+1}-\rho}{\rho+1}$.

Hence

$$
\frac{m_{2}\left(n_{k}\right)}{n_{k}}=\frac{1}{\rho^{2 k}-1} \frac{\rho^{2 k+1}-\rho}{\rho+1}
$$

which tends to 1 when $k \rightarrow \infty$.

The proof that the vertical Lyapunov exponent is $\log (\kappa \alpha)$ follows in a similar way as before.

To prove the last statement of the lemma note that

$$
\left|\operatorname{det}\left(\left.D f\right|_{A}\right)\right|=\frac{d}{\kappa \alpha} \kappa \alpha=d \quad \text { and } \quad\left|\operatorname{det}\left(\left.D f\right|_{B}\right)\right|=\frac{d}{\alpha} \alpha=d .
$$

Then, for every $x \in \Lambda$ (and in particular for every $x \in \widetilde{\Lambda}$ ),

$$
\frac{1}{n} \log \left|\operatorname{det}\left(D f^{n} x\right)\right|=\log (d) \text {. }
$$


Therefore,

$$
\limsup _{n \rightarrow \infty} \frac{1}{n} \log \left|\operatorname{det}\left(D f^{n} x\right)\right|=\lim _{n \rightarrow \infty} \frac{1}{n} \log \left|\operatorname{det}\left(D f^{n} x\right)\right|=\log (d) .
$$

3.4. A common definition of SNA. One of the usual definitions of SNA is the one given for instance in [12]. The authors define the graph of a measurable function $\varphi: D \subset \mathbb{S}^{1} \rightarrow \mathbb{R}$ to be an SNA for a quasiperiodically forced system of the form (1) if it has the following four properties:

(1) for almost every initial condition $\left(\theta_{0}, x_{0}\right) \in \mathbb{S}^{1} \times \mathbb{R}$,

$$
\lim _{n \rightarrow \infty}\left|\psi^{(n)}\left(\theta_{0}, x_{0}\right)-\varphi\left(\theta_{n}\right)\right|=0
$$

where $\psi^{(n)}$ denotes the second component of the $n$th iterate of the system (this follows from the fact that for almost every $\theta \in \mathbb{S}^{1}$ the vertical Lyapunov exponent on the graph of $\varphi$ is nonpositive),

(2) it is invariant: $\psi(\theta, \varphi(\theta))=\varphi(\theta+\omega)$ for every $\theta \in \mathbb{S}^{1}$,

(3) the Lyapunov exponent on the attractor is 0 , because the basis of the system is an irrational rotation,

(4) it cannot be extended to the graph of a continuous function in $\mathbb{S}^{1}$.

The first condition of this definition, which we will informally call the onedimensional approach, corresponds to the definition of attractor the authors consider, the third one is the definition of nonchaoticity, and the last one sets up the strangeness condition.

The main differences between the one-dimensional approach and the one we propose are the following: First, the invariant objects satisfying the above definition are not closed in contrast with any standard definition of attractor.

On the other hand, the notion of nonchaoticity of the one-dimensional approach only takes into account the dynamics on the graph of $\varphi$, whereas ours takes into account all Lyapunov exponents. Thus, the notion of nonchaoticity that we propose is stronger than the one contained in the onedimensional approach. However, the notion of strangeness considered in the one-dimensional approach is stronger than the one we propose.

Finally, the one-dimensional approach definition strongly depends on the fact that the system is a quasiperiodic skew product.

4. Some examples of SNA's. First of all notice that Keller's [17] model (see Example 1 and Theorem 2.1) has an SNA when $\sigma>1$ and $g$ vanishes at some point. Indeed, since $\varphi$ (the map giving the invariant graph) is discontinuous almost everywhere, its graph is not closed. So, a reasonable choice for the attractor is the closure of the graph of $\varphi$. This set is an attractor in view of Theorem 2.1(f), and the strangeness follows from Theorem 2.1(d) (see also statement (c)). Finally, the system is nonchaotic by Remark 3.4 
and because the vertical Lyapunov exponent exists for almost every $\theta$ and for every $x>0$, and it is negative.

Next we want to see that the Grebogi et al. [10] model (see Example 1) has an SNA. To do it we will prove that a larger class of models which are "two-sided" generalisations of the Keller model have an SNA, and we will obtain the desired result as a corollary.

The systems we will consider are of the form

$$
\left\{\begin{array}{l}
\theta_{n+1}=R_{\omega}\left(\theta_{n}\right), \\
x_{n+1}=f\left(x_{n}\right) g\left(\theta_{n}\right),
\end{array}\right.
$$

where $\left(\theta_{n}, x_{n}\right) \in \mathbb{S}^{1} \times \mathbb{R}, \omega \in \mathbb{R} \backslash \mathbb{Q}, g: \mathbb{S}^{1} \rightarrow \mathbb{R}$ is bounded, differentiable and vanishes at some point, and $f: \mathbb{R} \rightarrow \mathbb{R}$ is odd, bounded, $\mathcal{C}^{1}$ and satisfies $f(0)=0$. Moreover, either $f$ is increasing and $\left.f\right|_{(0, \infty)}$ strictly concave, or $f$ is decreasing and $\left.f\right|_{(0, \infty)}$ strictly convex.

As before, we define the parameter

$$
\sigma:=f^{\prime}(0) \exp \left(\int_{\mathbb{S}^{1}} \log |g(\theta)| d \theta\right)
$$

which is the Lyapunov exponent of the invariant circle $x=0$.

Then we have

Proposition 4.1. Assume that model (12) satisfies $\sigma>1$. Then it has an SNA.

Note that from Proposition 4.1 it follows immediately that the closure of the attractor of the Grebogi et al. [10] model is an SNA.

Proof of Proposition 4.1. Let $T(\theta, x):=\left(R_{\omega}(\theta), f(x) g(\theta)\right)$. Since $f$ and $g$ are bounded, there exists $Q$ such that $|f(x) g(\theta)| \leq Q$ for every $(\theta, x) \in$ $\mathbb{S}^{1} \times \mathbb{R}$. Then $T\left(\mathbb{S}^{1} \times[-Q, Q]\right) \subset \mathbb{S}^{1} \times[-Q, Q]$, and since $\mathbb{S}^{1} \times[-Q, Q]$ is compact, there is an attractor of the system $(12)$ in $\mathbb{S}^{1} \times[-Q, Q]$.

Now we consider the following related model:

$$
\left\{\begin{array}{l}
\theta_{n+1}=R_{\omega}\left(\theta_{n}\right) \\
z_{n+1}=|f|_{\mathbb{R}^{+}}\left(z_{n}\right)|| g\left(\theta_{n}\right) \mid
\end{array}\right.
$$

where $z_{n} \in \mathbb{R}^{+}$. This model satisfies the assumptions of the Keller model, and thus the conclusion of Theorem 2.1 holds for it. In particular, by using the notation of Theorem 2.1,

$$
\varphi(\theta)=|f|_{\mathbb{R}^{+}}\left(\varphi\left(R_{\omega}^{-1}(\theta)\right)\right)|| g\left(R_{\omega}^{-1}(\theta)\right) \mid .
$$

Then, since $f$ is odd,

$$
\begin{aligned}
\pm \varphi(\theta) & = \pm|f|_{\mathbb{R}^{+}}\left(\varphi\left(R_{\omega}^{-1}(\theta)\right)\right)|| g\left(R_{\omega}^{-1}(\theta)\right) \mid=( \pm s) f\left(\varphi\left(R_{\omega}^{-1}(\theta)\right)\right) g\left(R_{\omega}^{-1}(\theta)\right) \\
& =f\left(( \pm s) \varphi\left(R_{\omega}^{-1}(\theta)\right)\right) g\left(R_{\omega}^{-1}(\theta)\right)
\end{aligned}
$$


where $s:=\operatorname{sign}\left(g\left(R_{\omega}^{-1}(\theta)\right) \operatorname{sign}\left(f\left(\varphi\left(R_{\omega}^{-1}(\theta)\right)\right)\right)\right.$. Hence,

$$
\{\varphi(\theta),-\varphi(\theta)\}=f\left(\left\{\varphi\left(R_{\omega}^{-1}(\theta)\right),-\varphi\left(R_{\omega}^{-1}(\theta)\right)\right\}\right) g\left(R_{\omega}^{-1}(\theta)\right)
$$

and the closure of the graph of $\Phi(\theta)=\{\varphi(\theta),-\varphi(\theta)\}$ is an attractor for system (12). Moreover, since $\varphi$ is strange so is $\Phi$.

Finally, since $f$ is odd and the differences between systems (12) and (13) are essentially changes of sign in the coordinate $x$, it follows that the vertical Lyapunov exponents of both systems are the same. Thus, $\Phi$ is nonchaotic if and only if $\varphi$ is nonchaotic. Hence, system (12) has an SNA for $\sigma>1$ by Theorem 2.1.

The quasiperiodically quadratic family studied in [5] is another example of system with an SNA. It is immediate from [5, Main Theorem] that the closure of the invariant graph the author finds satisfies our definition.

4.1. One-dimensional SNA's. To end this section let us display a very different kind of SNA. Indeed, we will remark that absorbing Cantor sets and solenoidal attractors (of which the Feigenbaum attractor is a particular case) are, indeed, SNA's.

Let $f:[-1,1] \rightarrow[-1,1]$ be an $\mathcal{S}$-unimodal map (i.e. its Schwarzian derivative is negative wherever it is well defined) with a nonflat critical point $c$. By the topological classification of the attractors of these maps (see, for example, [9, Theorem 1.3 of Chapter V]), they have a unique attractor, which satisfies Milnor's definition (i.e. it is a metric attractor). More concretely, if $f$ has an attracting periodic orbit then the attractor is this periodic orbit; if $f$ is infinitely often renormalizable then the attractor is the Cantor set $\omega(c)$, which is called a solenoidal attractor. The last possibility is that $f$ is finitely often renormalizable. In this case the attractor is either a union of transitive intervals, or a Cantor set which is $\omega(c)$. In this last situation the attractor is called an absorbing Cantor set (or a wild Cantor attractor in [7]). The existence of this kind of attractor was proved in [6] and [7] under certain conditions (a particular case of maps satisfying these conditions are the Fibonacci maps). Whenever the attractor is a Cantor set, it is minimal and strange. Thus, to prove that it is an SNA we only have to see that the Lyapunov exponent is nonpositive for Lebesgue almost every point in $[-1,1]$. From statement 1 of the corollary on page 366 of [9], the Lyapunov exponent

$$
\lambda_{f}:=\limsup _{n \rightarrow \infty} \frac{1}{n} \log \left|D f^{n}(x)\right|
$$

exists for Lebesgue almost every $x$, and it is positive if and only if $f$ has an absolutely continuous invariant probability measure (with respect to the Lebesgue measure). By [9, Theorem 1.5 of Chapter V], if $f$ has an absolutely continuous invariant probability measure, then it is only finitely often renormalizable and it has no absorbing Cantor attractor. Thus, when the 
attractor of $f$ is solenoidal or an absorbing Cantor set, the Lyapunov exponent is less than or equal to zero for Lebesgue almost every $x$. Consequently, the attractor is an SNA in these cases.

Acknowledgements. The authors have been partially supported by the MEC grant numbers MTM2005-021329 and MTM2008-01486.

\section{References}

[1] Ll. Alsedà and M. Misiurewicz, Attractors for unimodal quasiperiodically forced maps, J. Difference Equations Appl. 14 (2008), 1175-1196.

[2] L. Barreira and Ya. B. Pesin, Lyapunov Exponents and Smooth Ergodic Theory, Univ. Lecture Ser. 23, Amer. Math. Soc., Providence, RI, 2002.

[3] L. Barreira and J. Schmeling, Sets of "non-typical" points have full topological entropy and full Hausdorff dimension, Israel J. Math. 116 (2000), 29-70.

[4] Z. I. Bezhaeva and V. I. Oseledets, On an example of a "strange nonchaotic attractor", Funktsional. Anal. i Prilozhen. 30 (1996), no. 4, 1-9, 95 (in Russian).

[5] K. Bjerklov, SNA's in the quasi-periodic quadratic family, Comm. Math. Phys. 286 (2009), 137-161.

[6] H. Bruin, Topological conditions for the existence of absorbing Cantor sets, Trans. Amer. Math. Soc. 350 (1998), 2229-2263.

[7] H. Bruin, G. Keller, T. Nowicki, and S. van Strien, Wild Cantor attractors exist, Ann. of Math. (2) 143 (1996), 97-130.

[8] R. de la Llave, private communication, 2006.

[9] W. de Melo and S. van Strien, One-Dimensional Dynamics, Ergeb. Math. Grenzgeb. 25, Springer, Berlin, 1993.

[10] C. Grebogi, E. Ott, S. Pelikan, and J. A. Yorke, Strange attractors that are not chaotic, Phys. D 13 (1984), 261-268.

[11] À. Haro, On strange attractors in a class of pinched skew products, preprint, 2007.

[12] À. Haro and J. Puig, Strange nonchaotic attractors in Harper maps, Chaos 16 (2006), no. 3, 033127, 7 pp.

[13] M. R. Herman, Une méthode pour minorer les exposants de Lyapounov et quelques exemples montrant le caractère local d'un théorème d'Arnol'd et de Moser sur le tore de dimension 2, Comment. Math. Helv. 58 (1983), 453-502.

[14] T. H. Jäger, Strange non-chaotic attractors in quasiperiodically forced circle maps, Comm. Math. Phys. 289 (2009), 253-289.

[15] - On the structure of strange non-chaotic attractors in pinched skew products, Ergodic Theory Dynam. Systems 27 (2007), 493-510.

[16] A. Jorba, J. C. Tatjer, C. Núñez, and R. Obaya, Old and new results on strange nonchaotic attractors, Int. J. Bifur. Chaos Appl. Sci. Engrg. 17 (2007), 3895-3928.

[17] G. Keller, A note on strange nonchaotic attractors, Fund. Math. 151 (1996), 139148.

[18] V. M. Millionščikov, Proof of the existence of irregular systems of linear differential equations with almost periodic coefficients, Differ. Uravneniya 4 (1968), 391-396 (in Russian).

[19] —, Proof of the existence of irregular systems of linear differential equations with quasi periodic coefficients, Differ. Uravneniya 5 (1969), 1979-1983 (in Russian).

[20] J. Milnor, On the concept of attractor, Comm. Math. Phys. 99 (1985), 177-195; Erratum, ibid. 102 (1985), 517-519. 
[21] T. Nishikawa and K. Kaneko, Fractalization of torus as a strange nonchaotic attractor, Phys. Rev. E 54 (1990), 6114-6124.

[22] A. Prasad, S. S. Negi, and R. Ramaswamy, Strange nonchaotic attractors, Int. J. Bifur. Chaos Appl. Sci. Engrg. 11 (2001), 291-309.

[23] C. Robinson, Dynamical Systems, 2nd ed., CRC Press, Boca Raton, FL, 1999.

[24] R. E. Vinograd, A problem suggested by N. P. Erugin, Differ. Uravneniya 11 (1975), 632-638 (in Rusian).

Lluís Alsedà, Sara Costa

Departament de Matemàtiques

Edifici Cc

Universitat Autònoma de Barcelona

08913 Cerdanyola del Vallès, Barcelona, Spain

E-mail: alseda@mat.uab.es

scosta@mat.uab.es

Received 18 February 2009;

in revised form 31 May 2009 